\title{
Pulmonary drug delivery
}

\begin{abstract}
"Pharmaceutical aerosol formulations are typically more sophisticated and less efficient than those for conventional routes of administration (e.g., oral and parenteral). Moreover, the lungs are complex in their anatomy and physiology so optimizing pulmonary delivery is challenging. Nevertheless, constant progress in this direction has been made..."
\end{abstract}

The respiratory tract has been used for centuries to deliver biologically active chemicals into the human body. Inhaled volatile oils and smoke from tobacco or other herbs have long been employed by ancient civilizations for therapeutic and recreational purposes [1]. This demonstrates the efficiency of the lungs, with a large surface area and profuse blood supply, to absorb drugs into the systemic circulation. Indeed, therapeutic proteins such as insulin and growth hormone have been delivered successfully by inhalation in modern times to treat systemic diseases. Inhalation is also the most effective route of administration for local diseases such as asthma, chronic obstructive pulmonary disease (COPD), and lung infections. The inhaled drug particles can target the site of action quickly with a relatively low dose, consequently decreasing systemic adverse effects. Pharmaceutical aerosol formulations are typically more sophisticated and less efficient than those for conventional routes of administration (e.g., oral and parenteral). Moreover, the lungs are complex in their anatomy and physiology so optimizing pulmonary delivery is challenging. Nevertheless, constant progress in this direction has been made, thus, it is appropriate to dedicate a focus issue on this topic.

The three editorials in this issue discuss various ways to improve pulmonary drug delivery. Connor and McDermott recognize that a successful pulmonary product is a complex interplay between the formulation, inhaler device and the patient [2]. Costly and inefficient traditional development paradigms of these products may be improved by adopting new regulatory strategies and clinical trial designs that have been established for pharmaceutical oral products. Onoue and Yamada explore the advantages of formulating pirfenidone, which is currently delivered orally for pulmonary fibrosis, into an inhalable form [3]. Inhaled pirfenidone is preferable to treat this condition due to its local targeting and has a better safety profile.
Lactose is the only approved carrier so far for dry-powder inhaler products. However, its variation in physicochemical properties, particle size and surface characteristics renders variability in the dispersion of micron sized drug particles. To address this issue, Nokhodchi and Larhrib explore using engineered mannitol particles with controlled characteristics to control the aerosol performance of the powder [4].

In the Ask the Experts feature, a panel of six distinguished pulmonary drug-delivery researchers discuss their experiences and outlook. Current marketed pharmaceutical aerosol products are inefficient compared with other dosage forms. Doubtlessly, they need to be improved but patient factors and affordability must also be taken into account during R\&D. The products should be user-friendly to encourage correct usage and compliance. The expert panel also point out the need for accurate in vitro-in vivo correlation and prediction of aerosol deposition in the future.

In the interview featured in this issue, Peter J Barnes gives his perspective on pulmonary delivery as a clinician. He believes that a personalized approach to treating asthma and COPD is more effective than prescribing the same therapy for all patients. He also recognizes the importance of patient education on inhaler usage and checks on their techniques. Barnes suggests that the ideal inhaled drug for inflammatory airway diseases can deposit in the peripheral lungs and be retained there to exert its anti-inflammatory effect. However, it should be readily broken down when it is absorbed systemically to minimize its exposure elsewhere in the body.

Salomon et al. report on the characterization of the in vitro permeability and cytotoxicity of CPZEN-45, a caprazamycin derivative that has previously been shown to be active against $\mathrm{Myco}$ bacterium tuberculosis [5]. Shur et al. investigated the effects of air-jet micronization, postmicronization conditioning and storage on the surface properties of budesonide particles [6]. In turn,

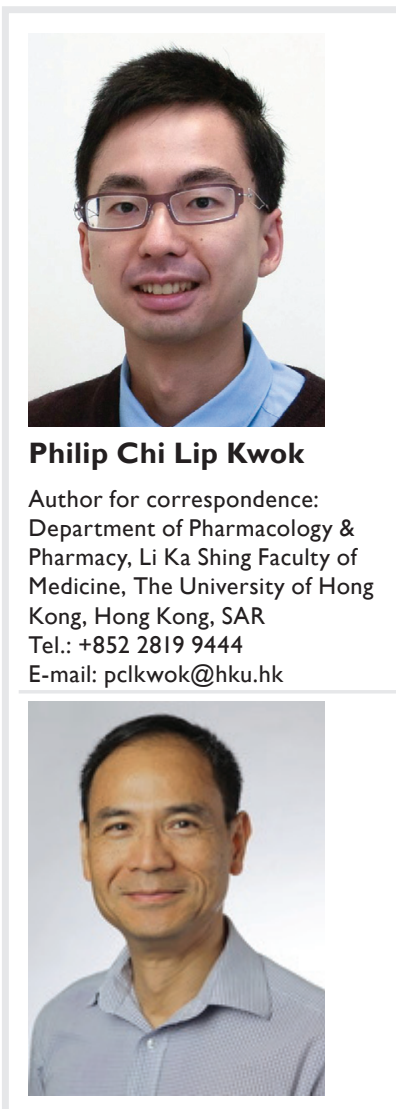

Hak-Kim Chan

Faculty of Pharmacy, The University of Sydney, Camperdown,

New South Wales 2006, Australia 
these affected the in vitro aerosol performance of the powders. In the special report, Smutney and Grant discuss various factors that can affect the performance of dry-powder inhalers [7]. These include the amount of powder being dispersed, the flow mechanics inside the inhaler, and the resistance of the inhaler device. Cipolla et al. review is on novel inhaled liposomal formulations [8]. Specifically, there are now at least two such products in late-stage clinical development, ARIKACE $^{\circledR}$ (liposomal amikacin; Insmed, Inc., Monmouth Junction, NJ, USA) and Pulmaquin ${ }^{\mathrm{TM}}$ (liposomal ciprofloxacin; Aradigm Corp., Hayward, CA, USA), both of which are indicated for pulmonary infections. The mechanisms of drug absorption and elimination from the lungs are reviewed by Ibrahim and GarciaContreras [9]. Due to the complex constitution of the airways and alveoli, investigation on these mechanisms is very difficult. The lungs also have physical and immunological defensive barriers that work against drug delivery. Thus, an understanding on the fate of drugs in this region is essential for facilitating pulmonary delivery of therapeutic agents. El-Gendy et al. review the treatment of respiratory diseases using exogenous surfactants, which have been extensively studied in animal models and clinical trials [10]. The history of surfactant therapy, the types of treatments, the development of new-generation surfactants and their mode of delivery are covered in this article. The electrostatics of pharmaceutical aerosols is a relatively new area of research but it is potentially important because it is one of the deposition mechanisms of particles in the airways. Significant advances in the past decade have improved understanding in this area. Wong et al. present an overview on this, with an emphasis on how electrostatic charge can be utilized to improve pulmonary drug delivery [11]. Finally, Mitchell and Nagel provide a critical review on the limitations of current pharmacopoeial methods for the in vitro testing of pharmaceutical aerosol products due to their oversimplifications of the clinical situation [12]. This has led to discrepancies in the in vitro-in vivo correlation of deposition data. Thus the in vitro testing methods need to be re-evaluated and modified to enhance their clinical relevance.

This issue offers a highlight on recent advances in pulmonary drug delivery. Challenges still remain but it is expected that solutions are possible with progresses in this field. The ultimate aim is to benefit the patients by optimizing drug delivery via the inhalation route.

\section{Financial \& competing interests disclosure}

The authors have no relevant affiliations or financial involvement with any organization or entity with a financial interest in or financial conflict with the subject matter or materials discussed in the manuscript. This includes employment, consultancies, honoraria, stock ownership or options, expert testimony, grants or patents received or pending, or royalties.

No writing assistance was utilized in the production of this manuscript.

\section{References}

1 Sanders M. Inhalation therapy: an historical review. Prim. Care Resp. J. 16(2), 71-81 (2007).

2 Connor A, McDermott J. Re-engineering the early development process for pulmonary drug delivery products. Ther. Deliv. 4(8), 883-885 (2013).

3 Onoue S, Yamada S. Pirfenidone in respirable powder form for the treatment of pulmonary fibrosis: a safer alternative to the current oral delivery system? Ther. Deliv. 4(8), 887-889 (2013).

4 Nokhodchi A, Larhrib E. Overcoming the undesirable properties of dry-powder inhalers with novel engineered mannitol particles. Ther. Deliv. 4(8), 879-882 (2013).
Salomon JJ, Galeron P, Schulte N et al. Biopharmaceutical in vitro characterisation of CPZEN-45, a drug candidate for inhalation therapy of tuberculosis. Ther. Deliv. 4(8), 915-923 (2013).

6 Shur J, Pitchayajittipong C, Rogueda P, Price R. Effect of processing history on the surface interfacial properties of budesonide in carrier-based dry-powder inhalers. Ther. Deliv. 4(8), 925-937 (2013).

7 Smutney CC, Grant M, Kinsey PS. Device factors affecting pulmonary delivery of dry powders. Ther. Deliv. 4(8), 939-949 (2013).

8 Cipolla D, Gonda I, Chan H-K. Liposomal formulations for inhalation. Ther. Deliv. 4(8), 1047-1072 (2013).
9 Ibrahim M, Garcia-Contreras L. Mechanisms of absorption and elimination of drugs administered by inhalation. Ther. Deliv. 4(8), 1027-1045 (2013).

10 El-Gendy N, Kaviratna A, Berkland C, Dhar P. Delivery and performance of surfactant replacement therapies to treat pulmonary disorders. Ther. Deliv. 4(8), 951-980 (2013).

11 Wong J, Kwok PCL, Chan H-K. Electrostatics in pharmaceutical aerosols for inhalation. Ther. Deliv. 4(8), 981-1002 (2013).

12 Mitchell JP, Nagel MW. Improved laboratory test methods for orallyinhaled products. Ther. Deliv. 4(8), 1003-1026 (2013). 